\title{
Surfactants identified in synovial fluid and their ability to act as boundary lubricants
}

\author{
B. A. HILLS AND B. D. BUTLER
}

From the Department of Anesthesiology, the University of Texas Medical School at Houston, Texas 77030, USA

SUMmaRY Thin-layer chromatography has been used to identify phospholipids extracted from canine synovial fluid, the major component (45\%) being phosphatidyl choline (PC). The extracts and their components have been shown to be surface active in reducing the surface tension of water and to be readily adsorbed to hydrophilic solids, whose surfaces then become hydrophobic. These adsorbed monolayers of synovial surfactant were then found to be excellent boundary lubricants in vitro, reducing the coefficient of kinetic friction $(\mu)$ in the dry state and under physiological loading by up to $97 \%$ for extracts and $99 \%$ for PC alone, reaching $\mu=0 \cdot 01$. Surface-active phospholipid is put forward as the possible active ingredient in joint lubrication and shown to be consistent with previous biochemical studies to elucidate its identity. The model essentially follows the classical Hardy model for boundary lubrication imparted by surfactants. It is discussed in relation to a new approach in providing artificial lubrication and facilitating tissue release in patients with arthritis.

The aetiology of idiopathic osteoarthritis remains obscure, and the possibility that this disease may have a mechanical origin has been reviewed in detail elsewhere. ${ }^{1-3}$ Although it has never been substantiated that degenerative joint disease is a "wear and tear' phenomenon, ${ }^{4}$ there is no doubt that joint stiffness is a significant clinical finding in patients with arthritic disease in general. ${ }^{5}$ This has been associated with a decreased ability of cartilage to slide over cartilage and synovial membrane in the capsule, which in turn raises the issue of the normal means of lubrication which has been compromised for friction to exceed normal levels.

Theories of joint lubrication have been comprehensively reviewed, ${ }^{23}$ from which it is probably fair to conclude that both of the primary categories of lubrication, namely, hydrodynamic ${ }^{6}$ and boundary, ${ }^{7}$ are operative at the articular surfaces. The hydrodynamic approach has diversified into many variations by which the bearing surface is supported by a fluid pressure rather than direct solid-to-solid contact, the "squeeze film, and 'weeping bearing", being two of the more interesting models. These models have

Accepted for publication 9 January 1984.

Correspondence to Dr B. A. Hills, Department of Anesthesiology, University of Texas Medical School at Houston. Texas 77030, USA. been appropriately termed 'hydrostatic' lubrication by Radin and Paul, ${ }^{10}$ who emphasise that, although they could well apply at high speeds, boundary lubrication would still be most desirable at low speeds. McCutchen ${ }^{3}$ points out that 'weeping' and boundary lubrication are complementary, since it is difficult to envisage hydrostatic support for a bearing surface without some solid-to-solid contact of the matrix material of the fluid-retaining 'pockets' on opposing surfaces. Boundary lubrication could also provide the second line of defence against friction and wear when fluid pressure fails for pathological reasons. In any case it is hard to discount boundary lubrication when joint friction is found to be largely independent of sliding speed ${ }^{7}$ and is lower than that of water even at zero speed. ${ }^{1}$ This poses the question of identifying the active ingredient.

Turning to the synovial fluid for evidence, we find this liquid is a good lubricant where soft surfaces can be deformed to spread the load more evenly and, presumably, reduce the load over areas of peak stress. ${ }^{11}$ The hyaluronic acid component of synovial fluid has certainly been shown ${ }^{5}$ to be a very effective lubricant with low-load bearing, but under other conditions hyaluronic acid does not lubricate at all. ${ }^{12}$ The question then remains as to what ingredient, if any, is likely to provide lubrication under the vital 
conditions of high-load bearing when any mucin layer or hydrodynamic film is more likely to be penetrated and allow asperities from one solid surface to make contact with the other. Moreover, the lubrication of weight-bearing joints is unaffected by hyaluronidase even though it reduces the viscosity of synovial fluid. ${ }^{13}$ This detraction from mucopolysaccharides as the active ingredient is further substantiated by the finding ${ }^{12}$ that the hyaluronicacid phase of centrifuged synovial fluid was inactive. This leaves proteins and lipids as two possibilities.

Radin et al. ${ }^{12}$ have implicated protein as the active ingredient in joint lubrication, because the lubricating qualities were found in the layer of centrifuged synovial fluid containing protein. This proteinaceous component could be the glycoprotein lubricating fraction defined by chemical analysis by Swann et al. ${ }^{14}$ or either of the two chemically distinct lipoprotein forms found in ox synovial fluid by Curtain. ${ }^{15}$ The lipoidal fraction of these forms has been analysed by Silpananta et al. ${ }^{16}$ and found to contain the usual range of lipids, including phospholipid. This is interesting because various surfaceactive phospholipids have been implicated as boundary lubricants in pleural movement, ${ }^{17}$ while the extraction procedure used by Swann et al. ${ }^{14}$ would seem unlikely to have excluded phospholipid as a contaminant in their lubricating glycoprotein fraction.

In pursuing the protein lead Linn and $\operatorname{Radin}^{13}$ have found that trypsin can inhibit lubrication of the major weight-bearing joints, though McCutchen ${ }^{1}$ raises the point that this could be attributed to digestion of cartilage even though an inhibitor was added. Trypsin could also denature lipoprotein. Since serum protein fails to display similar lubricating abilities, ${ }^{18}$ any active protein would need to be specific to synovial fluid. ${ }^{12}$

Even though a strong case can be made for protein as the active ingredient in synovial fluid, none of the major arguments cited above exclude phospholipid. On the other hand friction of the human hip joint was raised by $130 \%$ when the articular surfaces were rinsed with fat solvents, ${ }^{19}$ which incidentally were not solvents for protein. Phospholipids directly adsorbed to articular cartilage could provide its very slippery or 'oily' appearance as appropriately termed by McCutchen, ${ }^{3}$ who also points out the tenacious adhesion of the lubricant to the surface as though adsorbed. Arguments such as these raise the possibility that phospholipids should be included as a possible candidate for the active ingredient in synovial boundary lubrication, since the above lipid solvents should also remove phospholipids - even if directly adsorbed to the articular surface as found in other tissues. $^{2021}$ Moreover, phospholipids identified in the pleural space have been found to be very surface active, containing many of the same components as lung surfactant. ${ }^{19}$ When directly adsorbed to solid surfaces they act as very effective boundary lubricants, just as predicted from the basic theory of Hardy. ${ }^{22}$ In this regard it has been pointed out how phospholipid surfactants possess several molecular groupings conducive to their imparting good boundary lubrication, namely, adequate adsorption, straight fatty acid chains within the range of optimal length and good cohesion of the adsorbed layer to avoid its penetration by asperities on the counterface. ${ }^{17}$

Thus it would seem most pertinent to analyse synovial fluid for phospholipid composition and to test any such surfactants identified for their capability to act as boundary lubricants either individually or as an extract or a synthetic mixture simulating the extract. Tests of protein elimination by the extraction process are essential. This study uses thin-layer chromatography (TLC) as a standard method for separating any identified surface-active phospholipids and employs a standard test of kinetic friction under dry conditions ${ }^{23}$ to assess their capability to act as boundary lubricants under moderate loadbearing conditions.

\section{Materials and methods}

Surgical procedures. Sixteen dogs (11.9-24 kg) used in this study were anaesthetised with pentobarbital sodium (Nembutal, $30 \mathrm{mg} / \mathrm{kg}$ ) and then killed with saturated $\mathrm{KC} 1$ solution when prepared. An electrosurgical unit (Valley Laboratory) was used for tissue dissection to expose the capsule of the stifle joint (articulatis genus) and reduce the chances of contamination of the synovial fluid with blood. The above lying epidermal and muscle layers were sectioned at the level of the medial meniscus between the medial collateral ligament and the patellar ligament. A small opening $(1.5 \mathrm{~cm})$ was made through the joint capsular membranes for insertion of a 15 gauge Luer stub adapter connected to a sterile $10 \mathrm{ml}$ syringe. The synovial fluid was carefully aspirated from both stifle joints and transferred to acid-washed chloroform/acetonerinsed extraction tubes. Any sample showing contamination by blood was rejected. From eight animals an aliquot of fresh synovia was retained for the hyaluronic acid test and protein assay.

Lipid extraction procedures. The pooled synovia from both joints was centrifuged to remove any cellular debris, after which the lipid was extracted by the standard method of Folch et al. ${ }^{24} 25$ Essentially, 
the procedure consisted of a primary extraction with $20 \mathrm{ml}$ chloroform/methanol $(2: 1)$ followed by a salt solution ( $4 \mathrm{ml}$ of $0 \cdot 2 \mathrm{~N} \mathrm{KCl}$ in methanol) and then centrifuging at 3000 r.p.m. for $15 \mathrm{~min}$ at $18^{\circ} \mathrm{C}$. The lower chloroform phase was evaporated to dryness under $100 \%$ nitrogen followed by resuspension in a known amount of chloroform for further testing or analysis.

Hyaluronic acid test. The capillary turbidity (CT) test as described by Ibrahim and Streitfeld ${ }^{26}$ was used to derive a quantitative estimate of the hyaluronic acid from the stifle joint synovia both before and after the lipid extraction procedure. This test was used to verify that no hyaluronic acid remained in the lipid extract used as the lubricant in the friction test. Essentially, the procedure is based on the standard mucin clot (MC) $\operatorname{test}^{27}$ and the turbidometric reduction (TR) test. ${ }^{28}$

Protein assay. The total protein of the stifle joint synovia was determined before and after the lipid extraction procedure. The method used was the biuret assay (Gilford Diagnostics), whereby the peptide linkages undergo reactions with the cupric ions in an alkaline copper sulphate solution, yielding a violet colour which was read spectrophotometrically (Gilford 260) at $540 \lambda$.

Thin-layer chromatography. Two-dimensional thin-layer chromatography (TLC), as described in detail by Skipski et al., ${ }^{30}{ }^{31}$ was employed for the qualitative analysis of the phospholipid fraction obtained from the synovial extract. $50 \mu \mathrm{l}$ of the extracted fluid was spotted on $20 \times 20 \mathrm{~cm}$ precoated silica gel plates (Silica-gel $\mathrm{H}$ Analtech). The plates were then placed in solvent-saturated chambers with the phase I solution consisting of chloroform/ methanol/acetic acid/water (50:25:8:4), while the phase II solutions comprised the same components but in a ratio of 50:7.7:8:2. ${ }^{32}$ After separation the phospholipid spots were developed with a vapour spray of sulphuric acid $(38 \%)$ and formaldehyde $(37 \%)(97: 3)$ followed by heating at $180^{\circ} \mathrm{C}$ for 15 min.

Control standards of all the major phospholipid subgroups were spotted parallel to the rise of each phase to verify spot identification.

Phosphorus analysis. After each spot was circled and identified it was scraped off the TLC plate into an extraction tube and anlysed for its phosphorus content by the exact procedure described in detail by Rouser et al..$^{30}$ This technique essentially transposes phosphorus into a soluble compound which gives a colour reaction with ammonium molybdate and other reagents. The quantity of phosphorus is then determined by a spectrophotometer (Gilford260) at $797 \lambda$ following calibration with reagent blanks and silica controls taken from unspotted areas of the TLC plates. In a separate test of surfactant recovery from the TLC plates all values exceeded $95 \%$.

Friction test. The method adopted for assessing the ability of the surfactants to reduce friction between moving surfaces was a standard test ${ }^{23}$ used for the similar task of testing similar cationic surfactants as 'sizes' for textile fibres. This has been modified slightly ${ }^{17}$ to accommodate the slower speeds (36 r.p.m.) of slippage more appropriate to physiological movement. Any shearing of fibres would err on the conservative side by elevating $\mu$.

The test is based on measuring the force $(F)$ needed to prevent movement of a dry yarn passing over a dry rotating pulley on which it continually slips (Fig. 1). The coefficient of kinetic friction $(\mu)$ is then derived from the following equation ${ }^{32}$ :

$$
F / W=e^{\mu \theta}
$$

where $\theta$ is the angle (in radians) by which the yarn is diverted in passing over the pulley and $W$ is the counterweight.

The yarn used was cotton carboxylated according

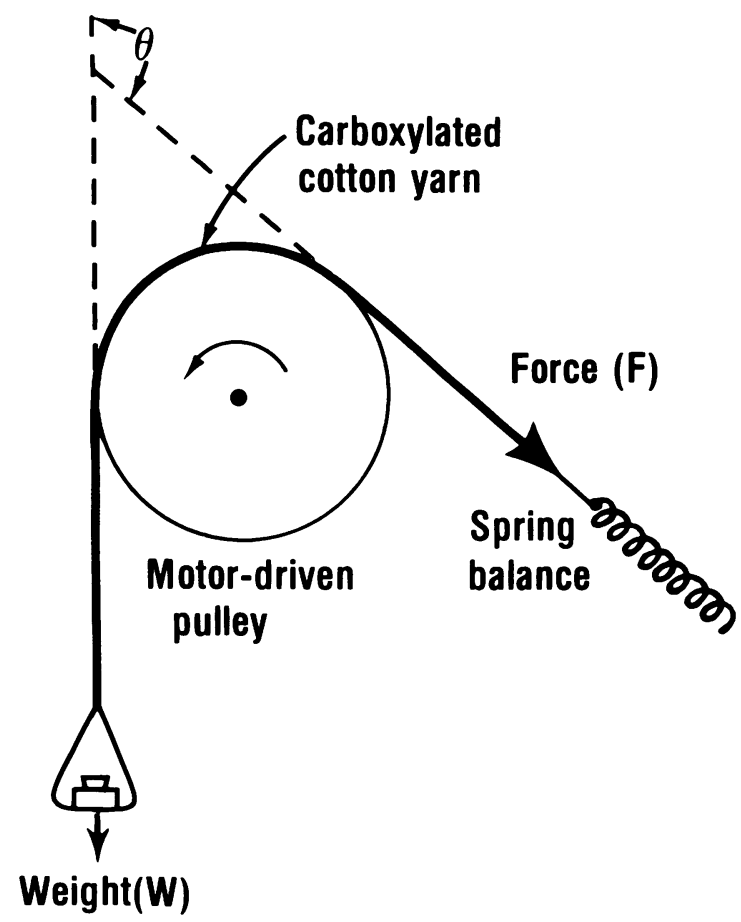

Fig. 1 Demonstrating the principle of the method ${ }^{2.3}$ for measuring the coefficient of kinetic friction $(\mu)$ for a dry fibre, with or without lubricant, passing over a slowly rotating glass pully on which it is continually slipping. The force $(\mathrm{F})$, weight $(\mathrm{W})$, and $\mu$ are related by equation 1 . 
to the standard method of Daul et al. ${ }^{33}$ with a known carboxyl content as determined by the standard method of Cumberbirch and Holker. ${ }^{34}$ In these tests the carboxyl content was $5.99 \%$, indicating that that percentage of the hydroxyl groups had been replaced by carboxyl groups. This was undertaken to simulate the situation which arises in all natural membranes, including synovial, where ionised carboxyl groups ${ }^{35}$ provide a prime site for the adsorption of cationic suractants ${ }^{36}$ - and zwitterions. $^{20} 37$

Surfactants. The surfactant was applied to the fibre by soaking the carboxylated cotton yarn in each extract and then allowing the chloroform to evaporate while control samples were soaked in chloroform only. Synthetic surfactants were also tested and these included: (1) a commercial mixture of natural phosphatidyl cholines (PC) - Sigma P8640; (2) sphingomyelins (Sp) - Sigma S7004; (3) a commercial mixture of natural phosphatidyl ethanolamines (PE) - Sigma 9137; (4) a commercial mixture of natural phosphatidyl inositols (PI) - Sigma EP-5766; (5) a commercial mixture of natural phosphatidyl serines (PS) - Sigma P8515; (6) a mixture of the above approximating to their relative preponderance in synovial extracts (Table 1), namely, 20:8:4:1:1, PC:Sp:PE:PI:PS. Each was made up to concentration of either $4 \mathrm{gm} / 1$ or $8 \mathrm{gm} / \mathrm{l}$ in chloroform and the solutions kept at $0^{\circ} \mathrm{C}$ under nitrogen.

Monoloayer deposition. The motor-drive pulley shown in Fig. 1 is a cylindrical glass tube $(1.5 \mathrm{~cm}$ diameter) on to which the same surfactant used to coat the yarn was deposited as an orientated monolayer according to the standard procedure of Blodget $\mathrm{t}^{38}$ as modified by Gaines. ${ }^{39}$ Its solution (1 $\mathrm{ml})$ was applied to the surface $\left(209 \mathrm{~cm}^{2}\right)$ of saline in a Langmuir trough, the solvent evaporating to deposit the surfactant, which was then compressed by $40 \%$ to form a monolayer. This was transferred to the immersed glass tube as it was slowly raised, while the movable barrier was further advanced to maintain constant surface tension. Glass surfaces

Table 1 Phospholipids identified in canine synovial fluid by thin-layer chromatography

\begin{tabular}{lc}
\hline Phospholipid & Percentage \pm SEM \\
\hline Phosphatidyl cholines (PC) & $44 \cdot 7 \pm 3 \cdot 90$ \\
Phosphatidyl ethanolamines (PE) & $15 \cdot 1 \pm 3 \cdot 25$ \\
Sphingomyelins (Sp) & $12 \cdot 2 \pm 2 \cdot 23$ \\
Lysophosphatidyl cholines (LPC) & $10 \cdot 9 \pm 2 \cdot 83$ \\
Phosphatidyl inositols (PI) & $7 \cdot 3 \pm 2 \cdot 08$ \\
Phosphatidyl serines (PS) & $7 \cdot 4 \pm 2 \cdot 14$ \\
\hline
\end{tabular}

Also trace amounts of phosphatidic acid and cardiolipin. were cleaned meticulously by standard procedures ${ }^{17}$ prior to coating.

Friction-test procedure. Each clean glass cylinder was placed in the friction tester with an uncoated carboxylated yarn as shown in Fig. 1 and the force $F$ measured three times by a force-displacement transducer (Grass Model 10D), the pulley being stopped between each measurement. The control yarn and glass cylinders were then coated with surfactant or extract as described above and the test repeated three times. The whole procedure of six measurements was repeated three to four times starting each time with a different glass cylinder and carboxylated cotton fibre - with three pulleys and three yarns per sample for 12-18 runs in total. The foregoing was repeated for each synovial extract, each component and the mixture of synthetics best approximating the natural composition (Table 1).

\section{Results}

THIN - LAYER CHROMATOGRAPHY

TLC analysis of synovial fluid from a total of eight dogs showed the presence of appreciable quantities of phospholipids. Spot identification based on comparison with synthetic standard phospholipids revealed the following groups of compounds: lysophosphatidyl choline (LPC), sphingomyelin (Sp), phosphatidyl cholines (PC), phosphatidyl inositols (PI), phosphatidyl serines (PS), and phosphatidyl ethanolamines (PE) (Table 1).

TESTS OF SURFACE ACTIVITY AND ADSORPTION The chloroform extraction procedure was found to leave no detectable amounts of hyaluronic acid (according to the CT tests) and no significant amount of protein $(<0.9 \mathrm{mg} / \mathrm{l})$ (according to the Biuret assay) in the phospholipid extracts for TLC or for the friction test.

The synovial extracts were found to give positive results for the two basic tests of surface activity:

(1) When applied to the surface of a Langmuir trough with a Wilhelmy balance to record surface tension - a standard procedure in pulmonary physiology $\mathrm{y}^{40}$ - surface tension $(\gamma)$ of the film was reduced to 45 dyne/cm and further reduced to 24 dyne/cm on compression of the film to $20 \%$ of its initial area. These values can be compared with 72 dyne/cm for water and $50 \mathrm{dyne} / \mathrm{cm}$ for serum. Typical first and third cycles are given in Fig. 2.

(2) While the above is a test of surfactant acting at a liquid surface, its activity at a solid surface was demonstrated by the contact angles of $55^{\circ}-61^{\circ}$ recorded when a drop of fluid was placed on monolayers of each synovial extract applied to a glass microscope slide by the same standard techni- 


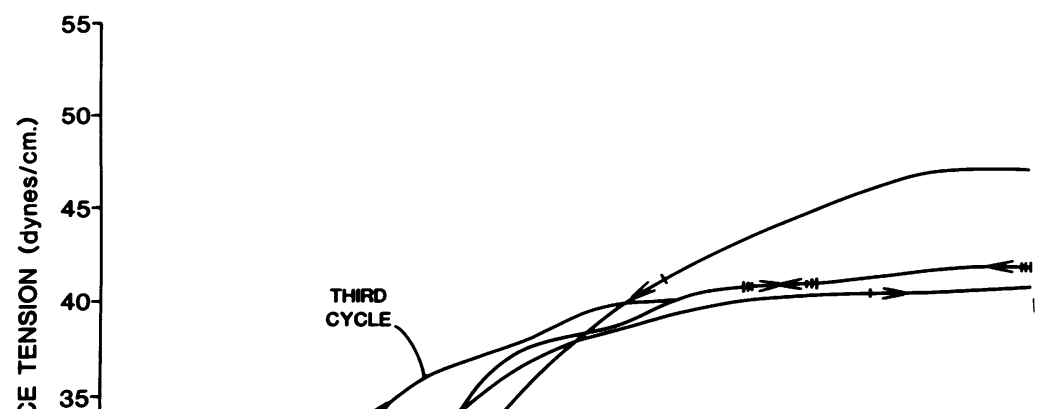

Fig. 2 The relationship between surface tension $(\gamma)$ and surface area (A) for a typical phospholipid extract from canine synovial fluid, the low $\gamma$ values and wide variations with A indicating high surface activity.

que used for depositing the monolayer in the friction test. A contact angle is induced when a surfactant is adsorbed to a hydrophilic solid surface such as glass to render it less wettable, its magnitude representing the degree of hydrophobicity for which typical values are $0^{\circ}$ for glass up to $108^{\circ}$ for Teflon. ${ }^{41}$ Contact angles were measured with a goniometer, as used previously ${ }^{42}$ to assess the hydrophobicity of surfaces.

\section{FRICTION TESTS}

In tests using both synovial extracts and commercially available samples of the identified phospholipids listed in Table 1 the force ( $F$ in Fig. 1) was

Table 2 Reduction in kinetic friction imparted by synovial phospholipids and their components

\begin{tabular}{|c|c|c|c|c|c|}
\hline \multirow[t]{2}{*}{ Sample } & \multicolumn{4}{|c|}{ Coefficient of kinetic friction $(\mu)$} & \multirow{2}{*}{$\begin{array}{l}\text { Percentage } \\
\text { reduction }\end{array}$} \\
\hline & Control $\pm S D$ & $n$ & $T e s t \pm S D$ & $n$ & \\
\hline Extract-dog 1 & $1.41 \pm 0.02$ & 10 & $0.25 \pm 0.02$ & 10 & 82 \\
\hline Extract-dog 2 & $1.49 \pm 0.07$ & 9 & $0.11 \pm 0.02$ & 14 & 93 \\
\hline Extract $\longrightarrow \operatorname{dog} 3$ & $1.44 \pm 0.03$ & 13 & $0.09 \pm 0.06$ & 14 & 94 \\
\hline Extract-dog 5 & $1.40 \pm 0.09$ & 9 & $0.27 \pm 0.05$ & 17 & 81 \\
\hline Extract-dog 6 & $1.46 \pm 0.06$ & 14 & $0.20 \pm 0.04$ & 19 & 86 \\
\hline Extract-dog 7 & $1.44 \pm 0.05$ & 11 & $0.13 \pm 0.07$ & 15 & 91 \\
\hline Extract-dog 8 & $1.49 \pm 0.07$ & 9 & $0.16 \pm 0.06$ & 14 & 89 \\
\hline Extract-dog 9 & $1.39 \pm 0.03$ & 11 & $0.04 \pm 0.01$ & 11 & 97 \\
\hline Extract-dog 10 & $1.49 \pm 0.02$ & 11 & $0.08 \pm 0.03$ & 5 & 95 \\
\hline Extract-dog 11 & $1.42 \pm 0.10$ & 13 & $0.15 \pm 0.02$ & 10 & 89 \\
\hline Extract-dog 12 & $1 \cdot 52 \pm 0.05$ & 14 & $0 \cdot 15 \pm 0 \cdot 03$ & 8 & 92 \\
\hline Commercial PC & $1.42 \pm 0.03$ & 24 & $0 \cdot 01 \pm 0 \cdot 00$ & 24 & 99 \\
\hline Commercial PE & $1.46 \pm 0.06$ & 24 & $0.62 \pm 0.06$ & 24 & 56 \\
\hline Commercial PS & $1.44 \pm 0.06$ & 24 & $0.57 \pm 0.03$ & 24 & 60 \\
\hline Commercial Sp & $1.40 \pm 0.03$ & 24 & $0.27 \pm 0.05$ & 24 & 81 \\
\hline Commercial PI & $1.40 \pm 0.04$ & 24 & $0.80 \pm 0.02$ & 24 & 43 \\
\hline Commercial PG & $1.56 \pm 0.04$ & 24 & $0.06 \pm 0.01$ & 24 & 96 \\
\hline Mixture* & $1.36 \pm 0.04$ & 24 & $0.19 \pm 0.05$ & 24 & 86 \\
\hline
\end{tabular}

${ }^{*}$ PC:PE:PS:Sp:PI = 20:5:1:14:4. 


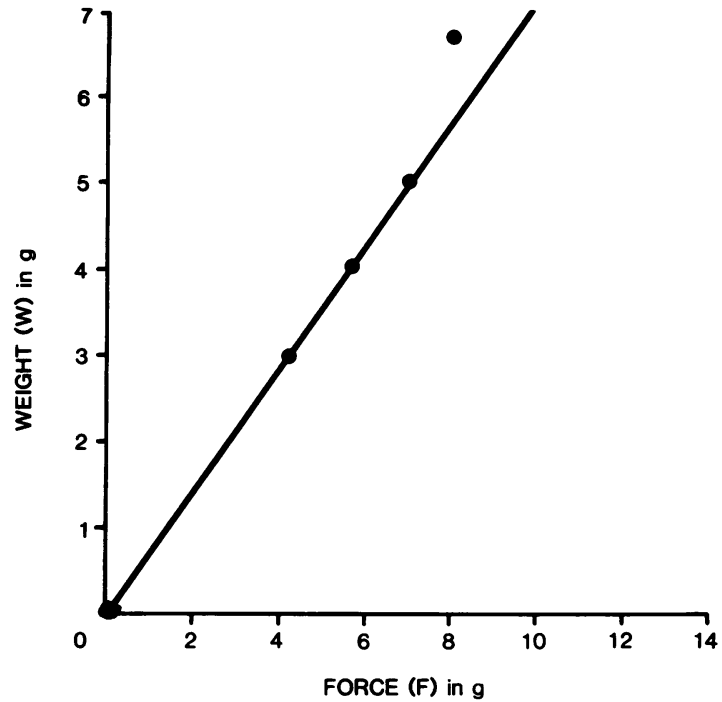

Fig. 3 The relationship between the force (F) and weight (W) when the carboxylated cotton yarn shown in Fig. 1 is lubricated with synovial extract for a fixed angle of deflection $(\theta)$ of $125^{\circ}$.

found to be directly proportional to the weight $(W)$, the angle $(\theta)$ being kept contant (Fig. 3). These results tend to confirm equation 1 , which was then used to evaluate the coefficient of kinetic friction $(\mu)$ for all synovial extracts (Table 2).

The reduction in $\mu$ averaged $90 \%$ in the synovial extracts, which exceeded that for most of the individual components except the major component (PC) for which the reduction reached $99 \%$. In all cases the reduction in $\mu$ was statistically significant according to the Student's $t$ test $(p>0 \cdot 0005)$.

\section{Discussion}

The TLC analysis of synovial fluid has identified many of the same groups of phospholipids found in many other body fluids and organs, ${ }^{43}$ while the subsequent phosphorus determinations have shown each to be present far in excess of the amount needed to give a monomolecular layer of that component in the joint. The major constituent is phosphatidyl choline (PC) (Table 1), although lower than in lung washings ${ }^{44}$ but comparable to the composition of pleural fluid in which the same surfactants have been implicated as effective boundary lubricants. ${ }^{17}$

The basic tests of surface activity used in this study showed that synovial extracts were effective in reducing the surface tension of water, especially on film compression, but not to the very low values often attributed to lung lavage extracts ${ }^{40}$ or their most active component - PC.

Teleologically this is compatible, since there is no air-fluid interface in the joint capsule, and any surface activity is more likely to be associated with solid surfaces to which surfactants can be directly adsorbed. Phospholipid adsorption to various surfaces has been demonstrated in vitro, including various synthetic surfaces, ${ }^{45}$ pulmonary and cutaneous epithelium, ${ }^{37}$ and the gastric mucosal lining. ${ }^{36}$ The contact angle measurements in this study indicate that the synovial extracts have about the same capibility of being adsorbed. When adsorption occurs, the surface must attract the hydrophilic moiety of the surfactant molecule, as depicted in Fig. 4, thus orientating the fatty acid chains outwards to give a hydrocardon layer which is easily detected as hydrophobic (Fig. 4). The acid polysaccharides incorporated into cartilage should provide ample negative sites for effective adsorption of the zwitterions - just as proposed ${ }^{21}$ for adsorption of essentially the same surfactants on to the mucins bound into the alveolar surface. ${ }^{46}$

The nature of the dipole in the zwitterion orientates the adsorbed molecule so that, when compacted with its neighbours, it produces a hydrocarbon outer surface (Fig. 4) with the ability to lubricate sliding against a similar surface much as one experiences when rubbing together two layers of polyethylene sheet. This invokes the original model of boundary lubrication proposed by Hardy. ${ }^{22}$ Load bearing is dependent upon cohesion of the adsorbed film, and it is interesting to note how this could be enhanced in the model in Fig. 4 by hydrogen bonding between adjacent phosphate groups or by placing calcium ions between them. ${ }^{21}$ This surface binding has been demonstrated for cations acting on phospholipid films at air-aqueous interfaces ${ }^{47}$ and is compatible with the commercial experience of adding phosphate to lubricants to improve their load-bearing capabilities ${ }^{48}$ Any compaction of the adsorbed monolayer by cohesive phosphate-tophosphate bonding is unlikely to be hindered by steric factors, since the cross-sectional areas of both hydrophilic and hydrophobic moieties is $40 \mathrm{~nm}^{2} .49$ At least, this applies to the saturated straight-chain fatty acids where straight are more effective than crooked chains, and two such chains per molecule are more effective than one. ${ }^{50}$

The molecular features of surface-active phospholipids described above and illustrated in Fig. 4 would indicate that such surfactants should be very good boundary lubricants. This is essentially reflected in the results given in Table 2 showing that phospholipid extracts from synovial fluid have the capability of reducing the coefficient of kinetic 


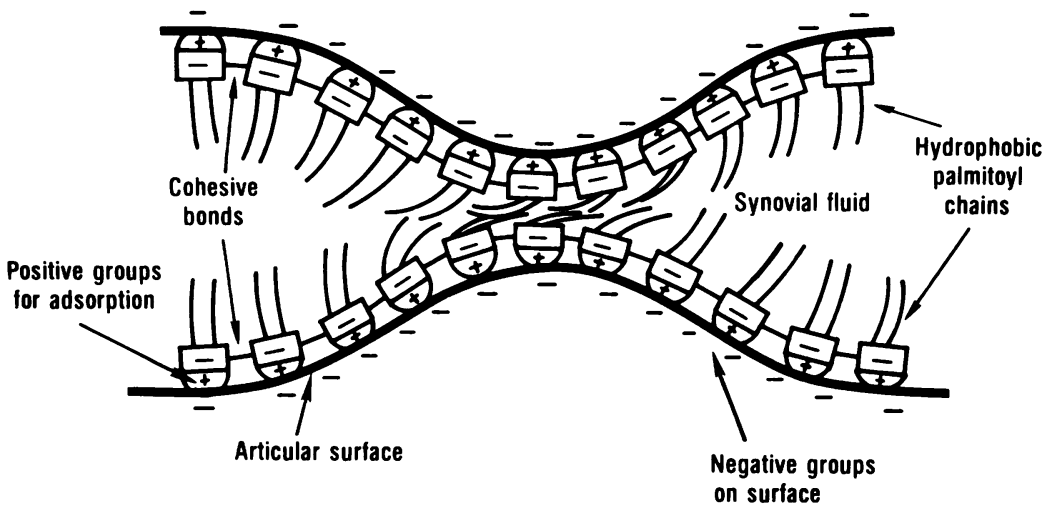

Fig. 4 General model of Hardy 22 for boundary lubrication imparted by surfactants modified ${ }^{77}$ to show two fatty acid chains per molecule and how the orientation of the electrical dipole of the zwitterion is ideal for its attraction to the fixed negative charges in synovial membrane to effect strong adsorption. Also indicated is possible hydrogen bonding between phosphatidyl groups to impart good cohesion necessary to prevent penetration of the adsorbed film (and wear) by asperities on the counterface. friction by about $90 \%$, while those from some dogs gives $\mu$ values as low as 0.04 which, for friction testing, is close to the value of 0.02 found for cartilage against cartilage ${ }^{51}$ under essentially wet conditions. Our study would be expected to give quite conservative values of $\mu$, since we are using dry conditions where there could be no contribution from hydrodynamic or hydrostatic lubrication. Moreover, the total absence of fluid would also hinder the replenishment of the boundary lubricant from any area which had been 'ploughed' - as it is termed $^{1}$ - by asperities from the counterface penetrating the adsorbed layer. It must also be remembered that the two hydrophilic solid surfaces used in this experiment, namely, glass and carboxylated cotton, need not adsorb the component surfactants in the same proportions as articular cartilage or synovial membrane, so that it is conceivable that, with the ability to replenish surfactant from micelles in a supernatant fluid, boundary lubrication imparted by surface-active phospholipids could reduce $\mu$ to the very low values of $0.005-0.024$ reported by Charnley. ${ }^{7}$ Even in our dry experiments the coefficient of friction for the major and most surface active constituent of synovial fluid, namely, PC, was reduced to about $0 \cdot 01$. This is still 4 -fold better than the lowest value for a solid $(0.04$ for Teflon) known to engineers. ${ }^{1}$ Moreover our studies were conducted at a mean control loading of $2.5 \mathrm{~kg} . \mathrm{cm}^{-2}$, which is in the upper physiological range of weight-bearing joints and yet below the limit of $4.5 \mathrm{~kg} . \mathrm{cm}^{-2}$ beyond which synovial fluid fails to lubricate. ${ }^{52}$

The above experiments do not prove that joint movement is normally lubricated by adsorbed phospholipids acting as boundary lubricants but they do indicate that these surfactants have the capability of doing so if adsorbed at articular surfaces in the same manner as they can be deposited in vitro. They could well be the active ingredient providing efficient boundary lubrication in conjunction with hydrostatic lubrication or as a back-up if the fluid were no longer retained in the pockets of the weeping bearing model and more solid-to-solid contact occurred. In this regard they would have another important secondary role as release agents, since, when adsorbed as depicted in Fig 4, the same phospholipids can reduce the adhesion of surfaces by about $90 \% .{ }^{53}$ It is tempting to speculate that the administration of synthetic phospholipids to pathological joints might help to prevent the binding of articular surfaces and to lubricate their movement.

The authors thank Mr Jim Romero and Miss Patricia Johnson for their assistance in collecting the data and Mrs Caroline Buggs Warner for her assistance in preparing this manuscript.

\section{References}

1 McCutchen C W. Lubrication of joints. In: Sokoloff M, ed. The joints and synovial fluid. New York: Academic Press, 1978: 438-83.

2 Sokoloff L. The biology of degenerative joint disease. Chicago: University of Chicago Press, 1969.

3 McCutchen $\mathrm{C} \mathrm{W}$. Lubrication of and by articular cartilage. In: Hall B K, ed. Cartilage. New York, Academic Press, 1983: 87-107.

4 Radin E L, Paul I L. Response of joints to impact loading. Arthritis Rheum 1971: 14: 356-62.

5 Radin E L, Paul I L. Swann D A. Schottstaedt E S. Lubrication of synovial membrane. Ann Rheum Dis 1971: 30: 322-5.

6 MacConaill M A. The function of intra-articular fibrocartilages, with special reference to the knee and inferior radio-ulnar joints. J Anat 1932; 66: 210-27.

7 Charnley J. Lubrication of animal joints. Inst Mech Eng. Proc Symp Biochem. London, 1959: 12-22.

8 Higginson G R. Norman R. The lubrication of porous elastic solids with reference to the functioning of human joints. $J$ Mech Eng Sci 1974; 16: 250-7.

9 McCutchen $\mathrm{C} \mathrm{W}$. Mechanism of animal joints. Spongehydrostatic and weeping bearings. Nature 1959; 184: 1284-5.

10 Radin E L. Paul I L. Joint function. Arthritis Rheum 1970; 13: 276-9.

11 McCutchen $\mathrm{C}$ W. Boundary lubrication by synovial fluid: demonstration and possible osmotic explanation. Fed Proc 1966; 25: 1061-8. 
12 Radin E L, Swann D A, Weisser P A. Separation of a hyaluronate-free lubricating fraction from synovial fluid. Nature 1970; 228: 377-8.

13 Linn F C, Radin E L. Lubrication of animal joint. III. The effect of certain chemical alterations of the cartilage and lubricant. Arthritis Rheum 1968; 11: 674-82.

14 Swann D A, Hendren R B, Radin E L, Sotman S L, Duda E A. The lubricating activity of synovial fluid glycoproteins. Arthritis Rheum 1981; 24: 22-30.

15 Curtain $\mathrm{C} \mathrm{C}$. The nature of the protein in the hyaluronic acid complex of bovine synovial fluid. Biochem $J$ 1955; 61: 688-97.

16 Silpananta P, Dunstone J R, Ogston A G. Protein associated with hyaluronic acid in ox synovial fluid. Aust J Biol Sci 1969; 22: 1031-7.

17 Hills B A, Butler B D, Barrow R E. Boundary lubrication imparted by pleural surfactants and their identification. $J$ Appl Physiol 1982; 53: 463-9.

18 Linn F C. Lubrication of animal joints. II The mechanism. $J$ Biochem 1968; 1: 193-205.

19 Little T, Freeman M A R, Swanson S A V. Experiments on friction in the human hip joint. In: Wright $\mathrm{V}$, ed. Lubrication and wear in joints. London: Sector, 1969: 110-16.

20 Hills B A. What is the true role of surfactant in the lung? Thorax 1981; 36: 1-4.

21 Hills B A. 'De-watering' capabilities of surfactants in human amniotic fluid. J Physiol (Lond) in press.

22 Hardy W B. Collected papers of Sir William Hardy. Cambridge: Cambridge U P, 1936.

23 British Standards Institute. British standards handbook Methods of test for textiles. London: HM Stationery Office 1974: 3/46-9.

24 Folch J, Ascoli I, Lees M, Meath J A, Lebaron F M. Preparation of lipide extracts from brain tissue. J Biol Chem 1951; 191: 833-41.

25 Folch J, Lees M, Sloane-Stanley G H. A simple method for the isolation and purification of total lipides from animal tissues. $J$ Biol Chem 1957; 226: 497-509.

26 Ibrahim A N, Streitfeld M M. The microassay of hyaluronic acid concentration and hyaluronidase activity by capillary turbidity (CT) and capillary turbidity reduction (CTR) tests. Anal Biochem 1973; 56: 428-34.

27 McClean D. Methods of assay of hyaluronidase and their correlation with skin diffusing activity. Biochem J 1943; 37: $169-77$.

28 Tolksdorf S, McCready M H, McCullagh D R, Schwenk E, Bloomfield $\mathrm{N} \mathrm{J}$. The turbidimetric assay of hyaluronidase. $J$ Lab Clin Med 1949; 34: 74-89.

29 Skipski U, Peterson R F, Sanders J, Barclay M. Thin-layer chromatography of phospholipids using silica gel without calcium sulfate binder. $J$ Lipid Res $1963 ; 4: 227-8$.

30 Rouser G, Siakotos A N, Fleischer S. Quantitative analysis of phospholipids by thin-layer chromatography and phosphorus analysis of spots. Lipids 1966; 1: 85-6.

31 Rouser G, Nelson G J, Fleischer S, Simon G. Lipid composition of animal cell membranes, organelles and organ. In: Chapman D, ed. Biological membranes. London: Academic Press, 1968.

32 Barmeister T, Avallone E A, Barmeister T (III). Mark's standard handbook for mechanical engineers. New York: McGraw-Hill, 1978: 3-31.

33 Daul G C, Reinhardt R M, Reid J D. Studies on the carboxymethylation of cotton. Textile Res $1952 ; 22$ : 787-92.

34 Cumberbirch R J E, Holker J R. Cerium-initiated polymerisation of some vinyl compounds in cellulose fibres. J Soc Dyers and Colourists 1966; 82: 59-63.

35 Davson H. A textbook of general physiology. London: Churchill, 1964.

36 Hills B A, Butler B D, Lichtenberger L M. Gastric mucosal barrier: the hydrophobic lining to the lumen of the stomach. Am J Physiol 1983; 7: G561-68.

37 Hills B A. Water repellency induced by pulmonary surfactants. J Physiol (Lond) 1982; 325: 175-86.

38 Blodgett $\mathrm{K}$ B. Films built by depositing successive monomolecular layers on a solid surface. J Am Chem Soc 1935; 57: 1007-22.

39 Gaines Jr, G L. Transfer of monolayers to solids. Multilayers. Insoluble monolayers at liquid-gas interfaces. New York: Wiley, 1966: 326-46.

40 Clements J A, Tierney D F. Alveolar instability associated with altered surface tension. In: Fenn W O, Rahn H, eds. Handbook of physiology: respiration. Washington: American Physiological Society 1965 : $1565-83$.

41 Adamson A W. Physical chemistry of surfaces. New York: Wiley, 1967: 352-67.

42 Hills B A. Contact hysteresis induced by pulmonary surfactants. J Appl Physiol 1983; 54: 420-6.

43 Montfoort A, Van Golde L M G, Van Deenen L L M. Molecular species of lecithins from various animal tissues. Biochem Biophys Acta 1971; 231: 335-42.

44 Frosonolo M F, Charms B L, Pawlowski R, Slivka S. Isolation, characterization and surface chemistry of a surface-active fraction from dog lung. $J$ Lipid Res 1970; 11: 439-57.

45 Gershfield N L. Selective phospholipid adsorption and atherosclerosis. Science 1979; 204: 506-8.

46 Meban C. An electron microscopic study of the acid mucosubstance lining the alveoli of hamster lung. Histochem J 1972; 4: 1-8.

47 Shah D O, Schulman J H. Binding of metal ions to monolayers of lecithins, plasmalogen, cardiolipin and dicetyl phosphate. $J$ Lipid Res 1965; 6: 341-9.

48 Larson C M, Larson R. Lubricant additives. In: O'Connor J J, Boyd J, eds. Standard handbook of lubrication engineering. New York: McGraw-Hill, 1968.

49 Harkins W D, Anderson T F. I: A simple accurate film balance of the vertical type for biological and chemical work and a theoretical and experimental comparison with the horizontal type. II: Tight packing of a monolayer by ions. J Am Chem Soc 1937; 59: 2189-97.

50 Evans W P. Cationic fabric softeners. Chem Industry 1969; 11: 893-903.

51 Jones E S. Joint lubrication. Lancet 1934; i: 1426-7.

52 McCutchen C W. More on weeping lubrications; experiments with Hydron, a microporous polyhydroxyalkylacrylic ester resin. In: Wright V, ed. Lubrication and wear in joints. London Sector, 1969: 117-23.

53 Hills B A. Analysis of Eustachian surfactant and its function as a release agent. Arch Otorhinolaryngol (NY) 1984; 110: 3-9. 